\title{
Interleukin-10 Expands Transit-Amplifying Cells While Depleting Lgr5+ Stem Cells via Inhibition of Wnt and Notch Signaling
}

\section{Fan Deng}

Southern Medical University Nanfang Hospital

Jingjuan Hu

Southern Medical University Nanfang Hospital

Xiao Yang

Southern Medical University Nanfang Hospital

Yifan Wang

Southern Medical University Nanfang Hospital

Kexuan Liu ( $\square$ liukexuan705@163.com )

Southern Medical University Nanfang Hospital https://orcid.org/0000-0003-0221-366X

Research article

Keywords: Intestinal Stem Cells, Interleukin-10, Mucosal healing, Wnt signaling, Notch signaling

Posted Date: July 22nd, 2020

DOl: https://doi.org/10.21203/rs.3.rs-44220/v1

License: (c) (i) This work is licensed under a Creative Commons Attribution 4.0 International License. Read Full License

Version of Record: A version of this preprint was published at Biochemical and Biophysical Research Communications on December 1st, 2020. See the published version at https://doi.org/10.1016/j.bbrc.2020.10.014. 


\section{Abstract}

Background \& Aims: Epithelial regeneration is essential for homeostasis and mucosal barrier repair. In infectious and immune-mediated intestinal diseases, interleukin (IL)-10 is thought to enhance these processes. We aimed to define the mechanism by which IL-10 played in mucosal healing or injury.

Methods: Intestinal stem cells (ISCs) cultures and mice were treated with recombinant mice IL-10 (rmIL10). The level of cell proliferation, differentiation, death and related signaling pathways for self-renewal of ISCs were measured in vitro and in vivo.

Results: It was uncovered that rmIL-10 increased the size and death, but reduced the total number of organoids. In addition, rmIL-10 depleted Lgr5 ${ }^{+}$ISCs and reduced epithelial proliferation, but enhanced the differentiation of epithelial cells and expanded numbers of transit-amplifying (TA) cells. These changes are related to the decrease of Wnt and Notch signals in vivo and in vitro. Meanwhile, increased expression of Paneth cells and decreased expression of enteroendocrine cells and goblet cells were induced by rmIL-10.

Conclusions: IL-10 reduces the survival of Lgr5 ${ }^{+}$ISCs and proliferation of epithelial cells by inhibiting Notch and Wnt signaling, but promotes enhanced the differentiation of epithelial cells and expanded numbers of TA cells. Therefore, IL-10 acts as an anti-inflammatory factor, but may damage intestinal mucosa repair and maybe a potential target for the treatment of intestinal injury.

\section{Introduction}

The intestine is not only the main place for digestion and absorption of nutrients, but also the innate barrier that guarantees the homeostasis of the body (1). On the one hand, the growth of the body requires the intestine to maintain a certain permeability to ensure that nutrients can be taken up and utilized to the maximum. On the other hand, the health of the body requires the intestine to maintain good tightness to prevent pathogenic bacteria and harmful substances such as toxins, to pass through the intestine and enter the body, thereby acting as the barrier (2). Intestinal mucosal barrier homeostasis and repair after injury depend on a tightly coordinated balance between self-renewal and differentiation of intestinal stem cells (ISCs) (1). The process is affected by both internal and external factors adjustment, comprising a signal produced by immune cells. However, little is known about the mechanisms that control the expansion of ISCs and transit-amplifying (TA) progenitor cells in response to tissue damage and immune activation.

Decades of research have produced a model of how ISC promotes homeostatic renewal and regeneration in the intestine. The current consensus is that small intestinal epithelial stem cells are composed of two types: +4 label-retaining cells (+ 4 LRCs) and crypt base columnar (CBC) cells. As active proliferating ISCs (aISCs), CBC cells constantly self-renew and generate all differentiated intestinal lineages, mediating the normal homeostatic turnover of the intestinal epithelium. However, + 4 LRCs, either as nonproliferating or slowly proliferating reserve ISCs (rISCs), usually perform regeneration tasks under tissue 
damage or extreme physiological stress conditions (3). The periodic renewal of intestinal epithelial cells depends largely on the self-renewal of ISCs, and gradually differentiates into mature intestinal epithelial cells along the villi axis. In addition, niche-signaling pathways that regulate the number of ISCs have been revealed by multiple studies using gene targeting in mice. Activation of Wnt/ $\beta$-catenin signaling is the main regulator of ISCs proliferation present in the base of normal intestinal crypts $(4,5)$. Notch signaling maintains the undifferentiated state of ISCs through "lateral inhibition", thereby playing an important role in the ISCs niche (4).

Secretory epithelial cells (goblet cells, Paneth cells, and enteroendocrine cells et al.) play an important role in the maintenance of mucosal homeostasis (6). Among them, Paneth cells can secrete antibacterial peptides and signal factors required for self-renewal of intestinal barrier homeostasis (7). Goblet cells can secrete mucus to lubricate the intestinal epithelium and prevent the colonization of harmful bacteria in the intestinal epithelium (8). Enteroendocrine cells can secrete a variety of hormones maintain the body's homeostasis and function (9).

Interleukin 10 (IL-10) is an anti-inflammatory cytokine with important immune regulation functions (10). Research shows that macrophage IL-10 signaling plays a key role in maintaining a regulated phenotype that prevents inflammatory bowel disease (IBD) (11). It has been revealed that resident intestinal bacteria activate the $B$ cells that produce IL-10 in the intestine, thereby reducing the activation of colon $\mathrm{T}$ cells and maintaining the homeostasis of the mucosa of the intestinal flora (12). Recent data indicate that IL-10 may promote ISCs self-renewal (13). However, the mechanisms by which IL-10 regulates intestinal diseases and the specific role of IL-10 on ISCs and mucosal homeostasis have not been fully identified.

Here, we sought to determine the effects of IL-10 on ISCs self-renewal and differentiation, as well as to gain a better understanding of the mechanisms that promote the relationship between epithelial recovery and healing with IL-10. For this purpose, we performed in vitro and in vivo experiments using ileum organoids or mice that were treated with recombinant murine IL-10 (rmIL-10) and screened its effect(s) on ISCs self-renewal and differentiation.

\section{Materials And Methods}

\section{Animal model}

All animal experiments comply with the regulations of the National Institutes of Health and were approved by committee of Southern Hospital of Southern Medical University. Male pathogen-free C57BL/6 mice, six to eight weeks old, were used in all experiments. Lgr5-EGFP-IRESCreER ${ }^{\mathrm{T} 2}$ mice were purchased from Shanghai Model Organisms Center, Inc. The mice were randomly divided into two groups. Control group: mice were intraperitoneally injected with $100 \mu \mathrm{PBS}(\mathrm{n}=8)$, Control + IL-10 group: rmlL-10 (Preprotech, Suzhou, China) was dissolved in PBS and administered to mice $(n=8)$ at a dose of $50 \mu \mathrm{g} / \mathrm{kg}$ via intraperitoneal injection for 5 days. Mice were anesthetized and euthanized. Blood and intestinal tissue were harvested in a sterile manner for further analysis. All mice were housed in a 12-hour 
light-dark cycle under controlled temperature and humidity conditions (temperature $25 \pm 1^{\circ} \mathrm{C}$; humidity 60 $\pm 5 \%$ ), and food and water were freely obtained.

\section{Crypt isolation, organoid culture, and quantification}

Small intestinal crypt isolation and culture were performed as previously described (14). Briefly, remove the mesentery, fat and blood vessels attached to the surface of the intestine, and the feces inside the intestine, cut the intestinal cavity after the longitudinal axis and cut the tissue into small pieces, then incubated in $5 \mathrm{mM}$ EDTA in PBS, on ice for $30 \mathrm{~min}$. Organoids are cultured in Matrigel ${ }^{\circledR}$ (Corning BioCoat, New York, USA) with IntestiCult ${ }^{\text {TM }}$ medium (STEMCELL Technologies Inc., Shanghai, China). The organoids were randomly divided into two groups. Normal control (NC) group, organoids were added $100 \mu \mathrm{l}$ PBS $(\mathrm{n}=6-8), \mathrm{NC}+\mathrm{IL}-10$ group: $\mathrm{rmlL}-10(5 \mathrm{ng} / \mathrm{mL})$ was dissolved in PBS and added to organoids $(n=6-8)$ for 5 days.

Image $\mathrm{J}$ software was used to calculate the number of organoids per well in different fields and planes. The increased number of organoids was calculated as the relative efficiency of organoid generation from ileum crypts.

\section{Calcein-AM staining to detect organoid survival}

The survival of organoids is done using a dead cell staining kit (BestBio, Shanghai, China), following the steps in the instructions. In short, after washing organoids twice with PBS, use Calcein-AM staining working solution to incubate in the dark at room temperature for 5 minutes, and capture the image with a fluorescence microscope (Olympus, Japan). Calculate the ratio of the number of Calcein-AM positive organoids to the total number of organoids and the intensity of Calcein-AM fluorescence.

\section{Reverse transcription-quantitative PCR (RT-qPCR)}

Total RNA was extracted using Trizol reagent according to the manufacturer's instructions. A reverse transcript enzyme (TOYOBO, Shanghai, China) was used to prepare cDNA according to the manufacturer's protocol. The real-time PCR reaction was performed using the ABI Q5 Real-Time PCR System with SYBR Green detection protocol (TOYOBO, Shanghai, China). The relative mRNA expression of target genes in mice was normalized to the house-keeping gene $18 \mathrm{~S}$, using the $2{ }^{-\wedge} \mathrm{C} C \mathrm{~T}$ method. The sequences of the main primers were shown in Table 1.

\section{Histological staining}

lleum tissue was collected and fixed in $4 \%$ paraformaldehyde. Then, the samples were embedded in paraffin and $5-\mu \mathrm{m}$-thick sections were sliced and stained with hematoxylin-eosin (HE) as previously described (15). The intestinal villus height and crypt depth were measured by automated image analysis in five randomly chosen 200X fields of each sample.

\section{Immunohistochemistry}


Paraffin sections (5- $\mu \mathrm{m})$ of the ileum tissue underwent appropriate heat-induced antigen retrieval and blocked as previously described (16). The following primary antibodies were used: rabbit anti-Notch1 antibody (Abcam, Shanghai, China), rabbit anti-beta Catenin antibody (Abcam), rabbit anti-Muc2 antibody (Proteintech, Wuhan, China), rabbit anti-Lyz1 antibody (Abcam) and rabbit anti-Chga antibody (Abcam). For negative controls, mouse or rabbit nonimmune serum, instead of the primary Abs, was used to treat tissue sections. Intensities quantification of Notch1, $\beta$-catenin, Muc2, Lyz1 and Chga staining was performed by automated image analysis in five randomly chosen 200X fields of each sample.

\section{Immunofluorescence}

Paraffin section samples were generated as above, blocked for $1 \mathrm{~h}$, and incubated overnight at $4{ }^{\circ} \mathrm{C}$ with rabbit anti-ki67 antibody (Abcam). Then wash the tissue with PBS, stain the nuclei with DAPI for 10 minutes, and capture the image with a fluorescence microscope (Olympus, Japan) at 200X.

Quantification of the fluorescence intensity of ki67 staining was performed by automated image analysis in five randomly chosen 200X fields of each sample.

\section{Statistical analysis}

Results are expressed as the mean \pm SEM. Statistical evaluations were performed using a two-tailed unpaired Student's t-test. A value of $P<0.05$ was considered a statistically significant difference. Statistics were calculated and display graphs were generated using Graphpad Prism 6.02.

\section{Results}

\section{Interleukin-10 expands the size of the ileum organoids but reduces its survival}

As shown in Fig. 1A, the ileum crypts of mice have a spherical structure after extraction, and they begin to bud on about 2-4 days, and form complex bud-like structures on 5-7 days. To better comprehend the effect of IL-10 on the mouse ileal epithelium, mouse ileum crypts were isolated and co-cultured with $5 \mathrm{ng} / \mathrm{ml} \mathrm{rmlL}-10$ in vitro. The medium with rmlL-10 notably reduced the efficiency of organoid generation, but increased the organoid size (Fig. 1B-D). Furthermore, rmlL-10 decreased the ratio of Calcein-AMlabeled live organoids/total organoids and the fluorescence intensity of Calcein-AM-labeled organoids (Fig. 1E-G). All these data indicate that rmIL-10 expands the size of the ileum organoids but reduces its survival.

\section{Interleukin-10 inhibits self-renewal of intestinal stem cells}

In order to observe the effect of IL-10 on the self-renewal of ISCs, the ileum crypts were isolated from Lgr5-EGFP-IRESCreER ${ }^{T 2}$ mice. The fluorescence intensity and proportion of organoids carrying EGFP fluorescent label in the untreated group were significantly stronger than those treated with rmIL-10 (Fig. 2A-C). Consistent with reduced Lgr5-EGFP ${ }^{+}$ISCs, the mRNA expression of alSCs marker Lgr5, Oflm4, 
and Sox 9 were lower in rmIL-10 treated organoids than control (Fig. 2D). In addition, rmIL-10 treatment resulted in a reduction in the mRNA of Ascl2, another alSCs marker, but it was not statistically significant (Fig. 2D). IL-10 not only reduces the expression of alSCs markers, the mRNA expression of rISCs markers Lrig1 was down-regulatd in rmIL-10 treated organoids, but the mRNA expression of other rISCs markers Bmi1, mTert, and Hopx did not obviously change in rmIL-10 treated organoids (Fig. 2E). Consistent with the results of organoids in vitro, the mRNA expression of alSCs markers Lgr5, Oflm4, Ascl2 and Sox9, and the mRNA expression of rISCs markers Lrig1, Bmi1, mTert, and Hopx were all reduced by rmlL-10 in mice (Fig. 2F-G). These data indicate that rmIL-10 inhibits self-renewal of ISCs, including alSCs and rISCs, in vitro and in vivo.

\section{Interleukin-10 reduces epithelium proliferation but expands transit-amplifying cells in vitro and in vivo}

The mRNA expression of ki67 (proliferation marker), Prom 1 (the stem and transit-amplifying cell marker), Zfp652 (transit-amplifying cell marker) and Press32 (immature enterocyte marker) were detected to assess the level of epithelial proliferation. As shown in Fig. 3, the mRNA expression of ki67 was lower, but the mRNA expression of Prom1, Zfp652 and Press32 were higher in rmlL-10 treated organoids and mice than them in untreated organoids and mice (Fig. 3A-B). Consistent with the mRNA expression of ki67, the protein expression of ki67 in mice was also decreased by rmlL-10 (Fig. 3C-D). Furthermore, rmlL-10 treatment significantly reduced the ileal villus height and the depth of crypts, but did not affect the pathological damage score (Fig. 3E-F). These data indicate that rmIL-10 reduces epithelium proliferation, but expands TA cells in vitro and in vivo.

\section{Interleukin-10 inhibits Wnt and Notch signaling}

Wnt/ $\beta$-catenin and Notch signaling are critical for ISCs maintenance and organoid function. Therefore, we observed whether rmIL-10 inhibits the Wnt/ $\beta$-catenin or Notch pathways that maintain intestinal stem cell activity. The results revealed that the mRNA levels of $W n t 3$, Wnt receptors $L r p 5$ and $L r p 6$, and that of the Wnt target gene $C$-myc were all lower in the rmlL-10 treated organoids (Fig. 4A) and mice (Fig. 4B) than in control groups. Similar effects were also observed in mice that rmlL-10 suppressed $\beta$-catenin protein expression as measured by immunohistochemistry (Fig. 4C). These data indicate that rmIL-10 inhibits ISCs Wnt signaling in vitro and in vivo.

Notch is closely related to Wnt in regulating the ISCs maintenance and ISCs differentiation direction. It was revealed that rmIL-10 treatment reduced transcription of Notch ligands Jagged1, and Hes1 as well as the Notch receptor Notch1 in organoids (Fig. 4D) and mice (Fig. 4E). Analogous effects were observed in the immunohistochemistry results in mice where rmlL-10 suppressed the Notch1 protein expression (Fig. 4F). These data indicate that rmIL-10 inhibits ISCs Notch signaling in vitro and in vivo.

\section{Interleukin-10 intermingles Epithelial Cell Differentiation}


The above-mentioned results show that IL-10 inhibited Igr5 ISCs numbers and epithelial proliferation; however, maintenance of the intestinal barrier homeostasis is inseparable from the presence of various intestinal epithelial mature cells. Therefore, we tested the expression of Paneth, enteroendocrine, and goblet cells to assess the level of differentiation of mature enterocytes. It was revealed that compared to control, rmlL-10-treated mice and ileum organoids expressed lower levels of the enteroendocrine cell markers Chga (Fig. 5A-D) and goblet cell markers Muc2 (Fig. 5E-H), but expressed higher levels of the Paneth cell markers $L y z 1$ (Fig. 5I-L) in vivo and in vitro. Taken together, these data indicate that rmIL-10 intermingle the differentiation of epithelial cells.

\section{Discussion}

Understanding how IL-10, released by various immune cells in the gastrointestinal tract, affects the selfrenewal of ISCs and the balance and homeostasis of intestinal epithelial proliferation and differentiation will help to understand the initiation, progress, and treatment of various gastrointestinal diseases, in which IL-10 is involved. In this study, we firstly demonstrated that IL-10 reduces the survival of Lgr5 ${ }^{+}$ISCs and proliferation of epithelial cells by inhibiting Notch and Wnt signaling, but promotes enhanced the differentiation of epithelial cells and expanded numbers of TA cells. In addition, this is novel finding that increased expression of Paneth cells and decreased expression of enteroendocrine cells and goblet cells were induced by rmIL-10. These suggest that IL-10 damages the self-renewal of ISCs, which is not conducive to the repair of intestinal mucosal injury.

Intestinal inflammation plays an important role in the repair and damage of intestinal mucosa, and IL-10, as a factor released directly by immune cells, plays its role in the direct interaction between immune cells and intestinal epithelium (17). IL-10 from different cells has been shown to perform multiple functions in a variety of intestinal diseases. In IBD, IL-10 signaling in macrophages, rather than in T cells, is essential for inducing $\mathrm{CD}^{206^{+}}$regulatory macrophages and anti-TNF therapeutic responses (11). Consistent with these results, it has been confirmed that intestinal IFN-y-producing type- 1 regulatory $T$ cells selectively down-regulated IL-10 in response to pro-inflammatory cytokines and may cause excessive intestinal inflammation in patients with IBD (18). In addition, this study showed that resident gut bacteria activated IL-10-producing B cells, which reduced colon T cell activation and maintained mucosal homeostasis to the gut bacteria (12). Short-chain fatty acids derived from microbiota can promote the production of IL-10 in Th1 cells, thereby maintaining intestinal homeostasis (10). Zhang et al. revealed that IL-10 could be a potential target for the damages of intestinal barrier and immunity in intestinal ischemia reperfusion (19). Furthermore, restoring normal bacteria through fecal microbiota transplantation (FMT) is considered a promising treatment for many diseases, and FMT activates immune cells to release IL-10 in order to suppress intestinal inflammation (20). Finally, bone marrow transplantation performed $24 \mathrm{~h}$ after total body irradiation improves intestinal barrier and stem cell recovery with induction of IL-10 and Notch signaling (21). All these indicate that IL-10 released by different immune cells plays an important role in maintaining intestinal homeostasis and anti-inflammatory. However, in this study, we firstly uncovered that IL-10 may play an adverse role in the process of intestinal mucosa repair and self-renewal of ISCs. 
A small group of Lgr5 ISCs divide regularly to produce highly proliferating progenitor cells, called TA cells, which eventually differentiate into an absorbable (enterocytes) or secretory (Paneth cells, goblet cells and enteroendocrine cells) cell lineage, while gradually moving up to the top of the villi (22). The close interaction between Lgr5 ISCs and Paneth cells is critical to maintaining the fate of Lgr5 ISCs, because Paneth cells provide crucial niche factors for self-renewal and differentiation of Lgr 5 ISCs (such as Wnt3a, BMP and Notch factors) (23). Paneth cells express Notch ligands, which bind to the Notch receptor on the Lgr5 ISCs to activate expression of downstream genes such as Hes 1 and Hes5. ISCs proliferation and maintenance are controlled by the Wnt/ $\beta$-catenin signaling pathway. Wnt ligands are released by Paneth cells and stromal cells (including macrophages, endothelial cells and neurons) located below the epithelial surface. Once released, Wnt ligands activate the low-density lipoprotein receptor-related protein 5/6 (Lrp5/6) and the frizzled protein co-receptors to facilitate $\beta$-catenin transport to the nucleus where it interacts with transcription factor 4 to maintain proliferation and differentiation of stem cells and epithelial cells. Here, we firstly demonstrated that IL-10 reduced ISCs survival and epithelial proliferation, and suppressed Notch and Wnt signaling. However, the number of Paneth cells was increased in IL-10 treated organoids and mice.

This study still has many limitations. This study only observed the effects of changes in IL-10 levels on intestinal epithelium cells and ISCs under normal conditions. However, we did not establish a specific disease model to observe the effects of changes in IL-10 levels on intestinal epithelium cells and ISCs. Therefore, the conclusion of this study may not be applicable to certain disease models. In addition, the signaling pathways that promote self-renewal of ISCs are not only Wnt and Notch, but also signaling pathways such as BMP. In this study, we did not observe the effect of IL-10 on other signaling pathways other than Wnt and Notch that promote self-renewal of ISCs. Meanwhile, we did not observe the effect of IL-10, an anti-inflammatory factor, on the inflammation level of ISCs and epithelial cells, and the number of macrophages and neutrophils etc.. Overall, we will establish a specific research model in future research to further explore the possibility and mechanism of IL-10 as a potential therapeutic target.

\section{Conclusion}

This study we showed that IL-10 inhibited self-renewal of ISCs and promoted intestinal epithelial cells to differentiate. Importantly, we identified that IL-10 inhibited the Wnt and Notch pathways that maintain the ISCs self-renewal. Our results are highlighting the function of IL-10 in inducing the proliferation of the TA cells supporting their role in ISCs differentiation while at the same time depleting the Lgr5 stem cells in order to maintain the homeostasis of the intestinal barrier and mucosal repair.

\section{Abbreviations}

\section{Declarations}

\section{Availability of data and materials}




\section{Ethics approval and consent to participate}

Not Applicable.

\section{Consent for publication}

All authors gave consent for publication.

\section{Competing interests}

The authors declare that they have no competing interests.

\section{Author Contributions}

Fan Deng, Jingjuan Hu and Kexuan Liu designed experiments, analyzed data and prepared this manuscript. Xiao Yang and Yifan Wang performed experiments and discussed the results. All authors reviewed and approved the submitted the manuscript.

\section{Synopsis}

IL-10 reduces the survival of $\mathrm{Lgr5}^{+}$intestinal stem cells and proliferation of epithelial cells by inhibiting Notch and Wnt signaling, but expanded numbers of transit-amplifying cells. This reveals that IL-10 may involve in intestinal mucosa repair and injury.

\section{Acknowledgement}

This work was supported by grants from National Natural Science Foundation, Beijing, China (81671955 to Kexuan Liu, 82902010 to Jingjuan Hu), Key Program of National Natural Science Foundation, Beijing, China (81730058 to Kexuan Liu). In addition, we thank Editage for the language editing and retouching services.

\section{Grant Support}

This work was supported by grants from National Natural Science Foundation, Beijing, China (81671955 to Ke-Xuan Liu, 82902010 to Jing-Juan Hu), Key Program of National Natural Science Foundation, Beijing, China (81730058 to Ke-Xuan Liu). 


\section{References}

1. Metidji A, et al. The Environmental Sensor AHR Protects from Inflammatory Damage by Maintaining Intestinal Stem Cell Homeostasis and Barrier Integrity. Immunity. 2018;49:353-62 e355.

2. Pan F, et al. Predominant gut Lactobacillus murinus strain mediates anti-inflammaging effects in calorie-restricted mice. Microbiome. 2018;6:54.

3. Bankaitis ED, Ha A, Kuo CJ, Magness ST. (2018) Reserve Stem Cells in Intestinal Homeostasis and Injury. Gastroenterology.

4. Zha JM, et al. Interleukin 22 Expands Transit-Amplifying Cells While Depleting Lgr5(+) Stem Cells via Inhibition of Wnt and Notch Signaling. Cellular molecular gastroenterology hepatology. 2019;7:25574.

5. Li B, et al. Impaired Wnt/beta-catenin pathway leads to dysfunction of intestinal regeneration during necrotizing enterocolitis. Cell death disease. 2019;10:743.

6. Lee YS, et al. Microbiota-Derived Lactate Accelerates Intestinal Stem-Cell-Mediated Epithelial Development. Cell Host Microbe. 2018;24:833-46 e836.

7. Date S, Sato T. Mini-gut organoids: reconstitution of the stem cell niche. Annu Rev Cell Dev Biol. 2015;31:269-89.

8. Olsen $\mathrm{MV}_{\text {, }}$ et al. (2020) Maresin 1, a specialized proresolving mediator, stimulates intracellular $[\mathrm{Ca}(2+)]$ and secretion in conjunctival goblet cells. Journal of cellular physiology.

9. Beumer J, Gehart H, Clevers H. (2020) Enteroendocrine dynamics - New tools reveal hormonal plasticity in the gut. Endocrine reviews.

10. Sun M, et al. Microbiota-derived short-chain fatty acids promote Th1 cell IL-10 production to maintain intestinal homeostasis. Nature communications. 2018;9:3555.

11. Koelink PJ, et al (2019) Anti-TNF therapy in IBD exerts its therapeutic effect through macrophage IL10 signalling. Gut.

12. Mishima $\mathrm{Y}$, et al. Microbiota maintain colonic homeostasis by activating TLR2/MyD88/PI3K signaling in IL-10-producing regulatory B cells. J Clin Invest. 2019;130:3702-16.

13. Biton $\mathrm{M}$, et al. T Helper Cell Cytokines Modulate Intestinal Stem Cell Renewal and Differentiation. Cell. 2018;175:1307-20.e1322.

14. Capeling $M$, et al. Generation of small intestinal organoids for experimental intestinal physiology. Methods Cell Biol. 2020;159:143-74.

15. Hu J, et al. Enteric dysbiosis-linked gut barrier disruption triggers early renal injury induced by chronic high salt feeding in mice. Exp Mol Med. 2017;49:e370.

16. He X, et al. Kindlin-2 deficiency induces fatal intestinal obstruction in mice. Theranostics. 2020;10:6182-200.

17. Miguel Quiros, et al. Resolvin E1 is a pro-repair molecule that promotes intestinal epithelial wound healing. Proc Natl Acad Sci USA. 2020;117(17):9477-82. 
18. Alfen JS, et al. Intestinal IFN-gamma-producing type 1 regulatory T cells coexpress CCR5 and programmed cell death protein 1 and downregulate IL-10 in the inflamed guts of patients with inflammatory bowel disease. J Allergy Clin Immunol. 2018;142:1537-47 e1538.

19. Zhang XY, Guan S, Zhang HF, Li RY, Liu ZM. Activation of PD-1 Protects Intestinal Immune Defense Through IL-10/miR-155 Pathway After Intestinal Ischemia Reperfusion. Digestive diseases sciences. 2018;63:3307-16.

20. Burrello $\mathrm{C}$, et al. Therapeutic faecal microbiota transplantation controls intestinal inflammation through IL10 secretion by immune cells. Nature communications. 2018;9:5184.

21. Wei L, et al. (2018) The GS-nitroxide JP4-039 improves intestinal barrier and stem cell recovery in irradiated mice. Scientific reports 8: 2072.

22. Lee JS, et al. AHR drives the development of gut ILC22 cells and postnatal lymphoid tissues via pathways dependent on and independent of Notch. Nature immunology. 2011;13:144-51.

23. Serra D, et al. (2019) Self-organization and symmetry breaking in intestinal organoid development. Nature.

\section{Tables}

Due to technical limitations, the tabes are provided in the Supplementary Files section.

\section{Figures}


A

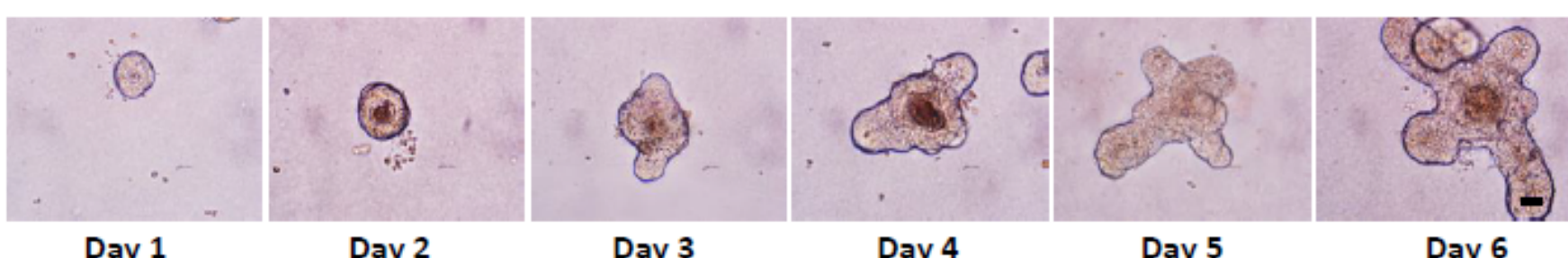

Day 1

Day 2

Day 3

Day 4

Day 5

Day 6

B

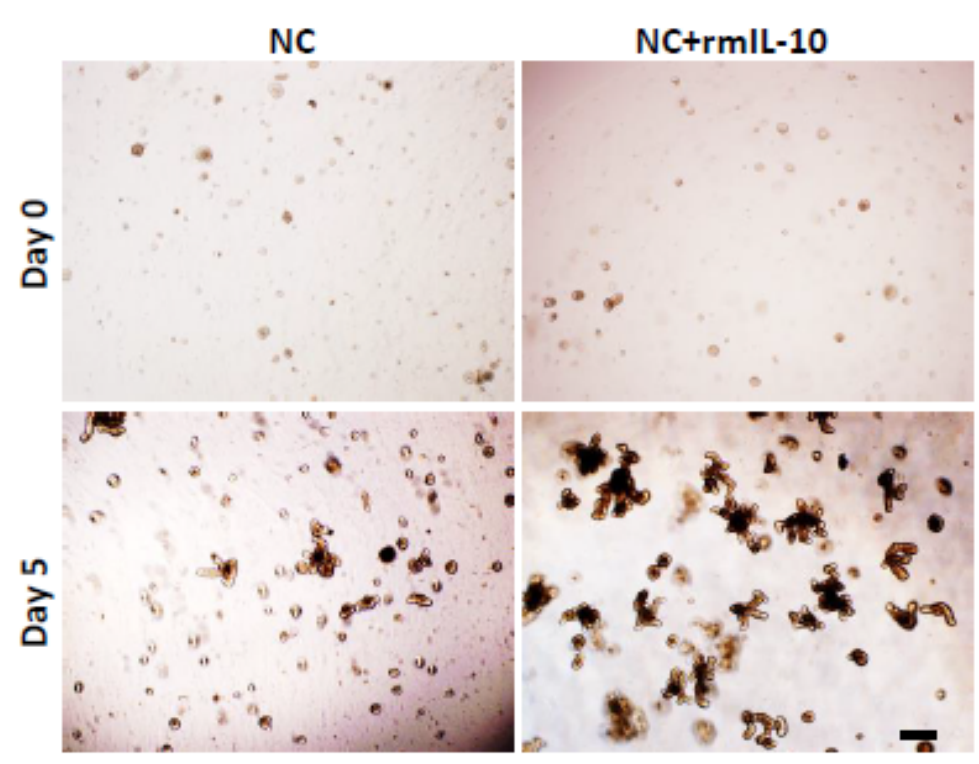

E

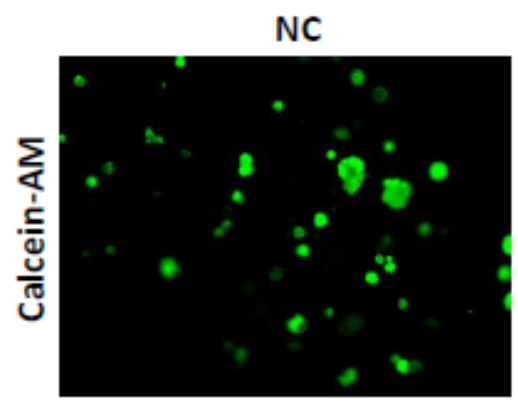

NC+rmIL-10

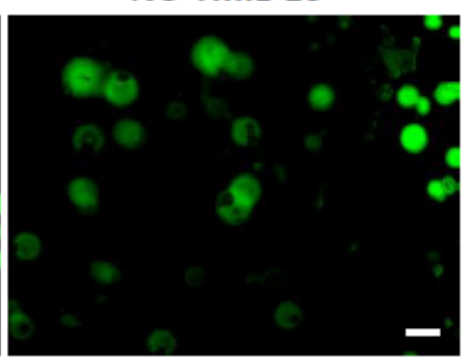

C

D
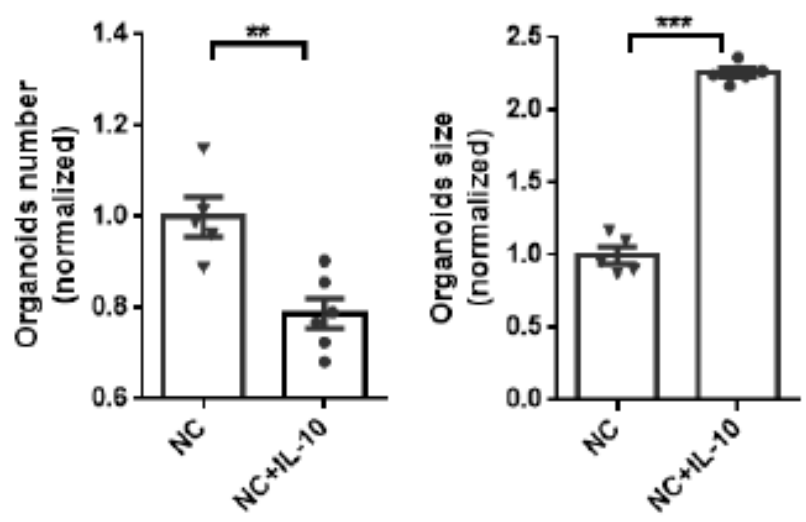

G

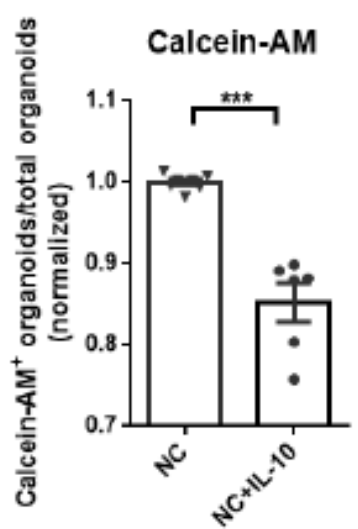

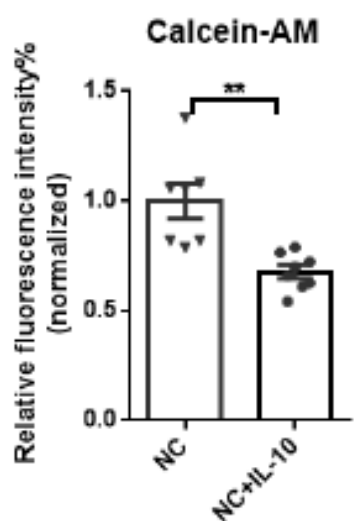

Figure 1

IL-10 expands the size of the ileum organoids but reduces its survival. (A) The growth state of organoids

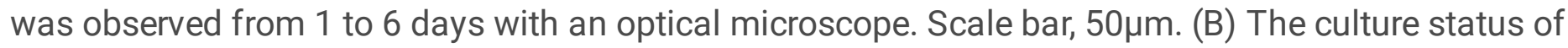
control-treated or rmIL-10-treated organoids on day 0 and day 5. (C) The number of organoids. (D) The sizes of organoids. (E) Calcein-AM live cells staining was used to detect organoid survival, scale bar, $50 \mu \mathrm{m}$. (F-G) The ratio of Calcein-AM labeled live organoids/total organoids and the fluorescence intensity of Calcein-AM labeled organoids. C57BL/6 mice were treated with or without rmIL-10 for 5 days $(n=8)$. Organoids were cultured for 5 days followed by treatment with the vehicle or with rmlL-10 (5 ng/ml) (n = 
6-8). The results are expressed as the mean \pm SEM. $n=8$. ${ }^{*} p<0.05,{ }^{*} p<0.01, * \star * p<0.001$ by twotailed Student's t test.

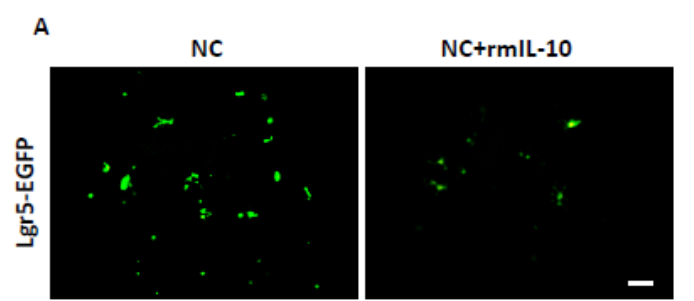

D
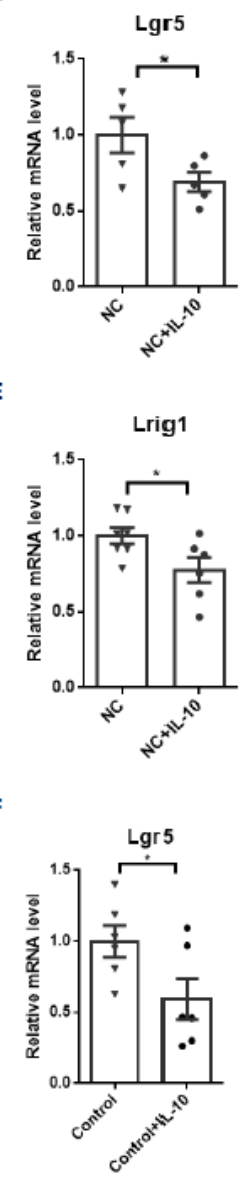

G

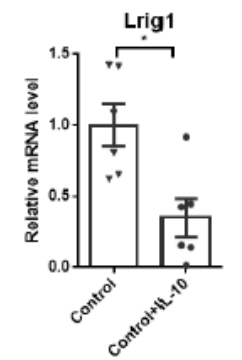

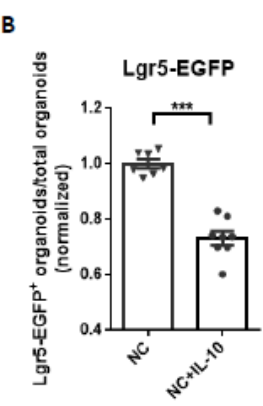
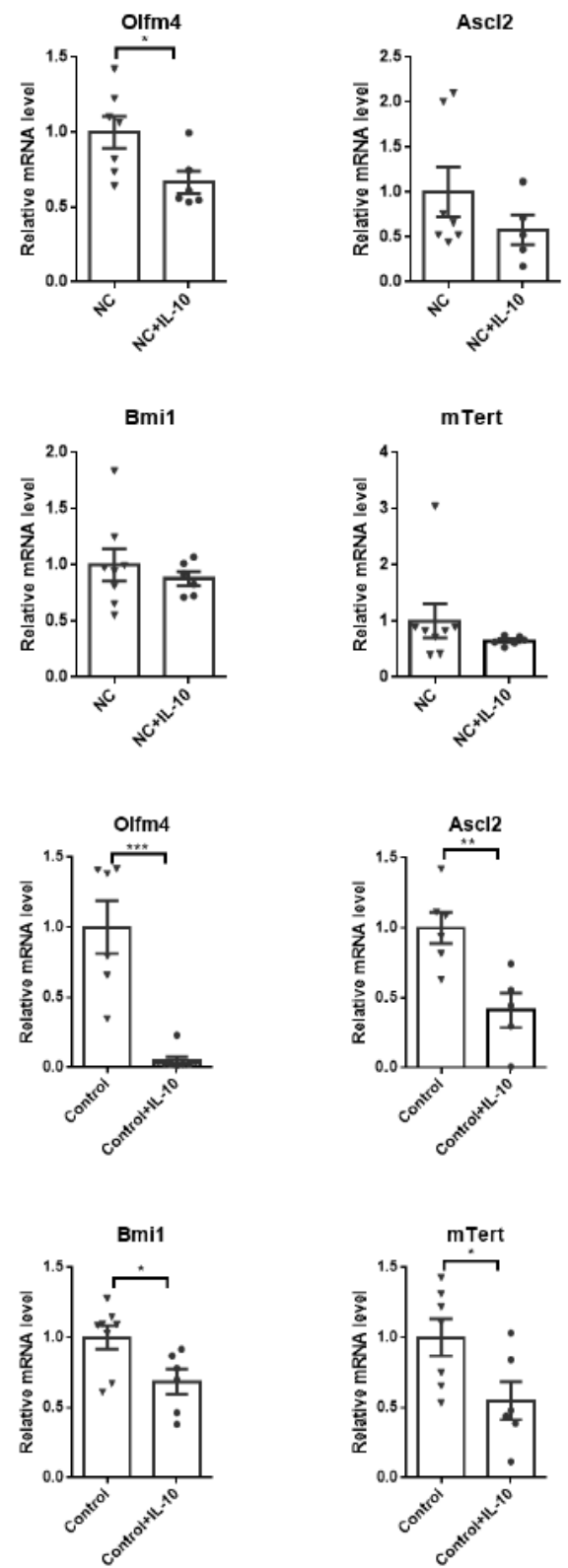

c
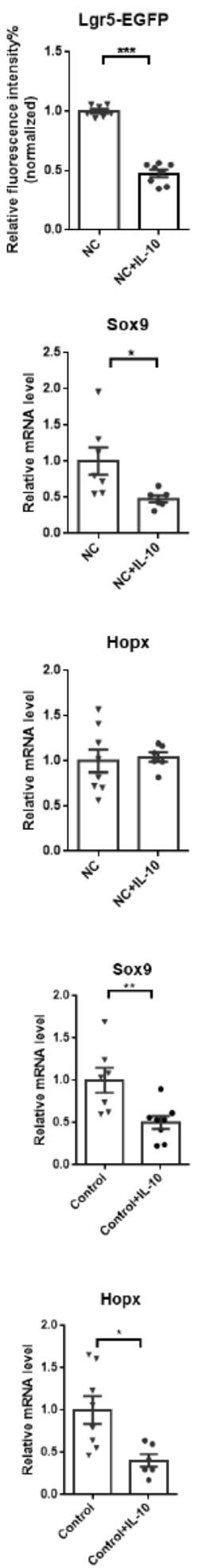

Figure 2

IL-10 inhibits self-renewal of intestinal stem cells. (A) The Lgr5-EGFP fluorescence of organoids, isolated from Lgr5-EGFP-IRESCreERT2 mice, detected by fluorescence microscope, scale bar, $50 \mu \mathrm{m}$. (B-C) The fluorescence intensity and proportion of Lgr5-EGFP organoids. (D) The mRNA expression of alSCs marker 
Lgr5, Oflm4, Ascl2 and Sox9 in organoids. (E) The mRNA expression of rISCs marker Lrig1, Bmi1, mTert and Hopx in organoids. (F) The mRNA expression of alSCs marker Lgr5, Oflm4, Ascl2 and Sox9 in mice. (G) The mRNA expression of rISCs marker Lrig1, Bmi1, mTert and Hopx in mice. C57BL/6 mice were treated with or without rmlL-10 for 5 days $(n=8)$. Organoids were cultured for 5 days followed by treatment with the vehicle or with $\mathrm{rmlL}-10(5 \mathrm{ng} / \mathrm{ml})(\mathrm{n}=6-8)$. The results are expressed as the mean \pm SEM. $n=8 .{ }^{*} p<0.05, * * p<0.01, * * * p<0.001$ by two-tailed Student's $t$ test. $A^{-}$
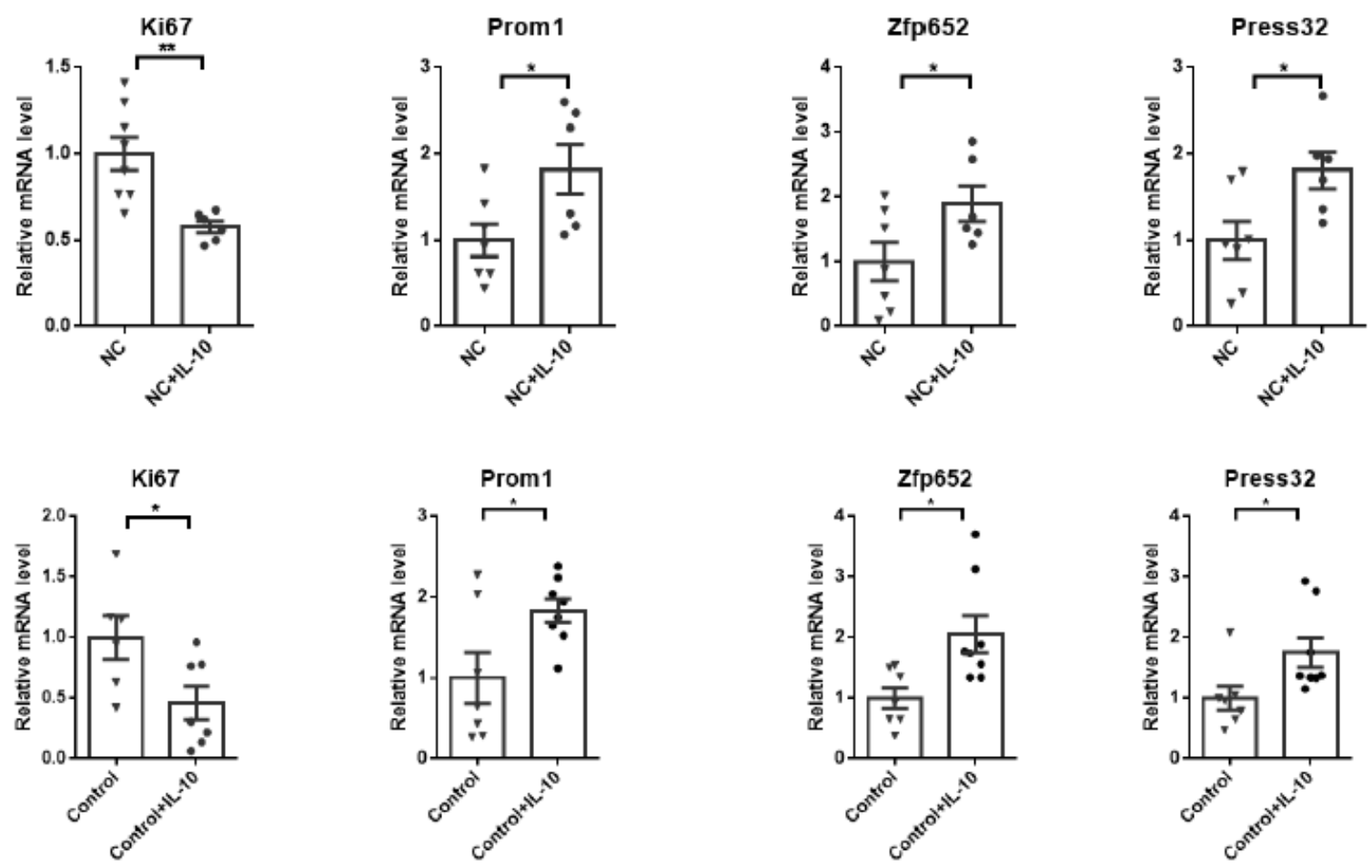

C

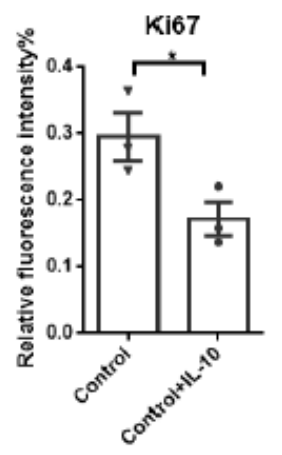

$\mathbf{E}$

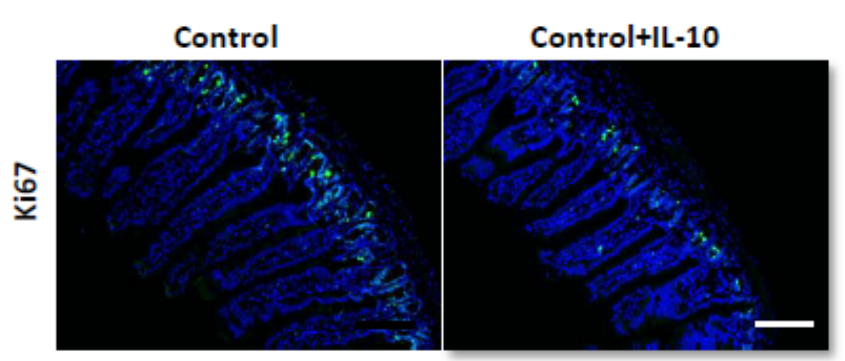

$\mathbf{F}$
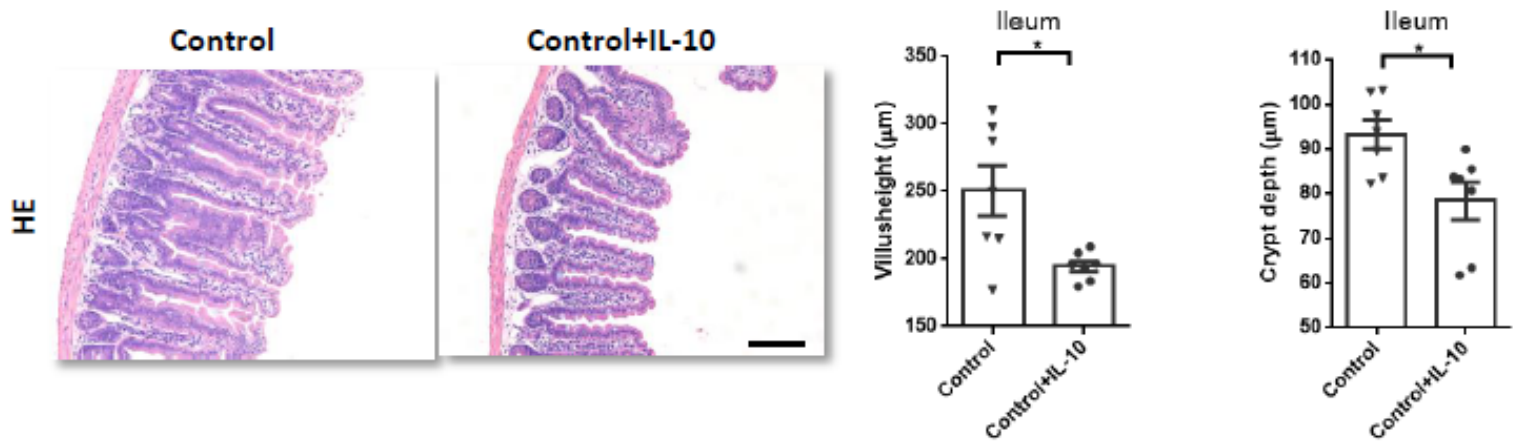

Figure 3 
IL-10 reduces epithelium proliferation but expands transit-amplifying cells in vitro and in vivo. (A-B) The mRNA expression of proliferation marker ki67, the stem and transit-amplifying cell marker Prom1, transitamplifying cell marker Zfp652 and immature enterocyte marker Press32 in organoids and mice. (C-D) The protein expression of ki67 in the ileum measured by immunofluorescent staining and quantification analysis. scale bar, $100 \mu \mathrm{m}$. (E-F) HE staining of ileum sections and quantitative analysis of intestinal villus height and crypt depth. scale bar, $100 \mu \mathrm{m}$. C57BL/ 6 mice were treated with or without rmIL-10 for 5 days $(n=8)$. Organoids were cultured for 5 days followed by treatment with the vehicle or with rmIL-10 ( 5

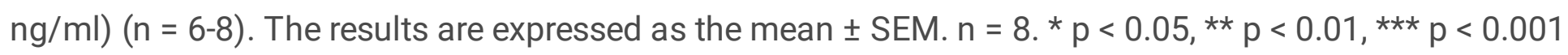
by two-tailed Student's t test. 

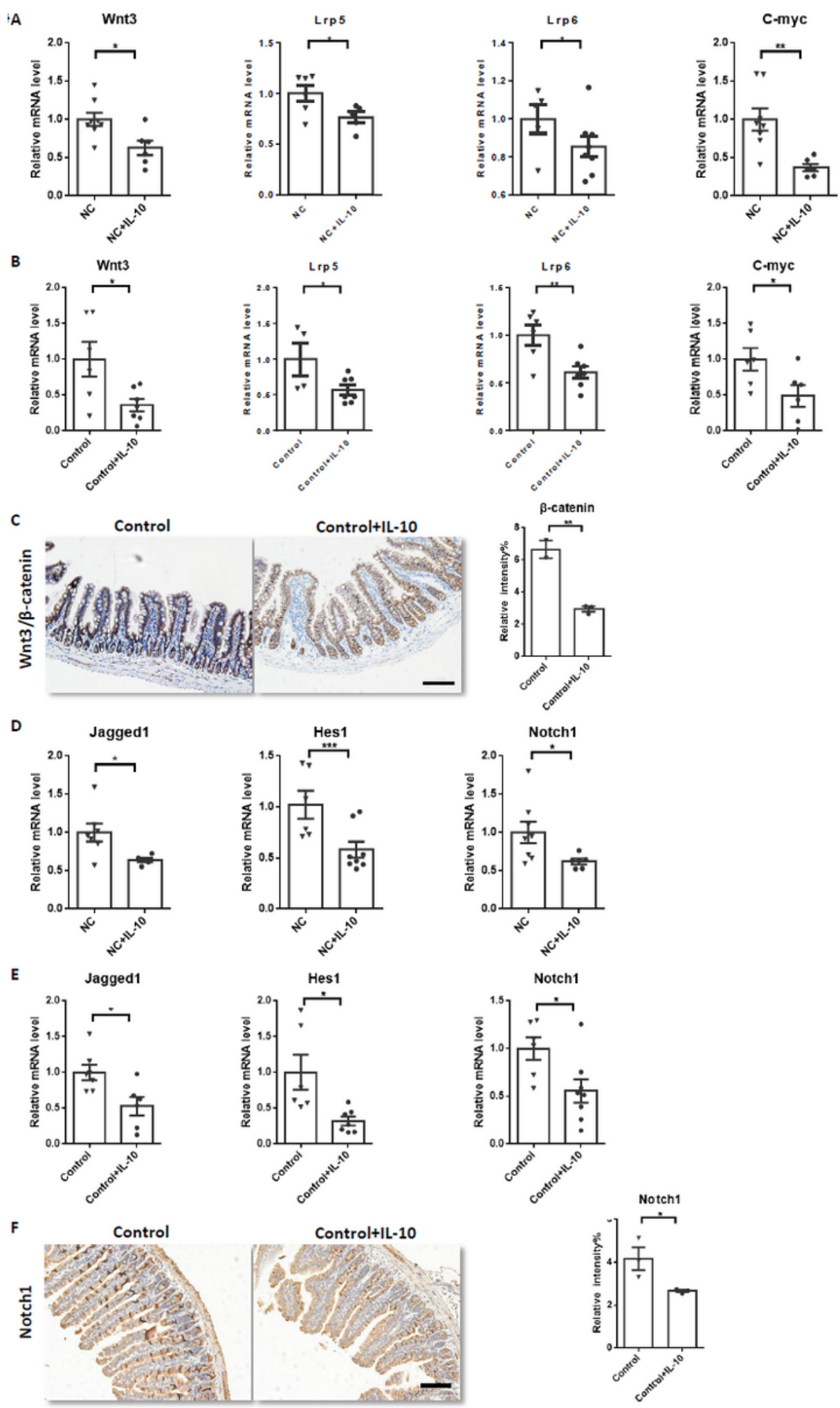

Figure 4

IL-10 inhibits Wnt and Notch signaling. (A-B) The mRNA expression of Wnt3, Wnt receptors Lrp5 and Lrp6, and Wnt target gene C-myc in organoids and mice. (C) The relative protein level of $\beta$-catenin in the ileum

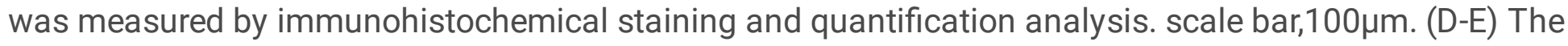
mRNA expression of Notch ligands Jagged1, Hes1 and the Notch receptor Notch1 in organoids and mice. (F) The relative protein level of Notch1 in the ileum was measured by immunohistochemical staining and 
quantification analysis. scale bar, $100 \mu \mathrm{m}$. C57BL/ 6 mice were treated with or without rmlL-10 for 5 days $(n=8)$. Organoids were cultured for 5 days followed by treatment with the vehicle or with rmIL-10 (5 $\mathrm{ng} / \mathrm{ml})(n=6-8)$. The results are expressed as the mean \pm SEM. $n=8 .{ }^{*} p<0.05,{ }^{* \star} p<0.01,{ }^{\star * \star} p<0.001$ by two-tailed Student's $t$ test.

A

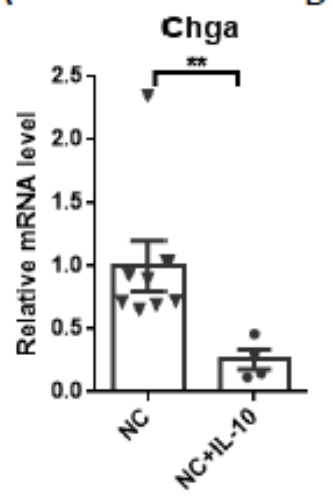

E

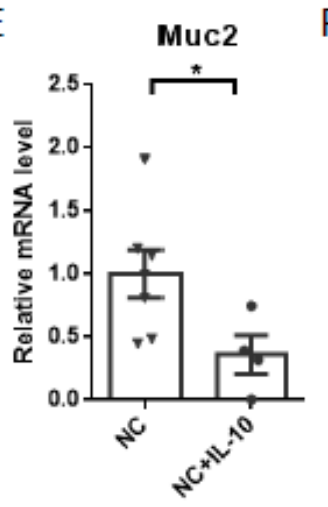

I

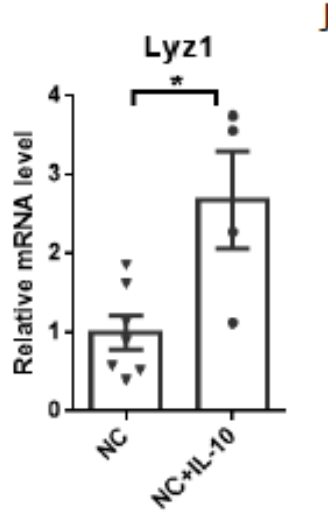

B

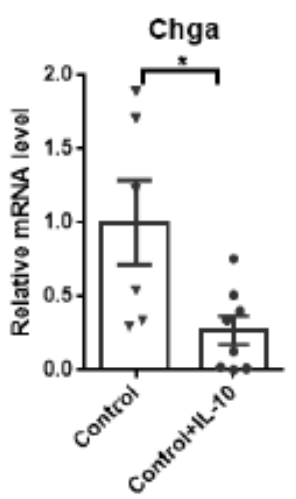

F

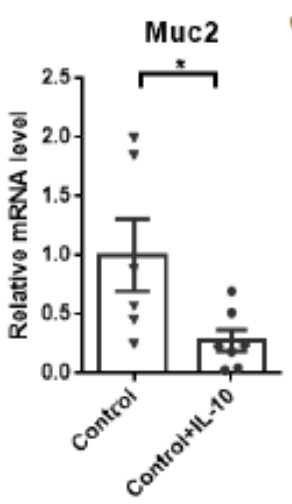

J

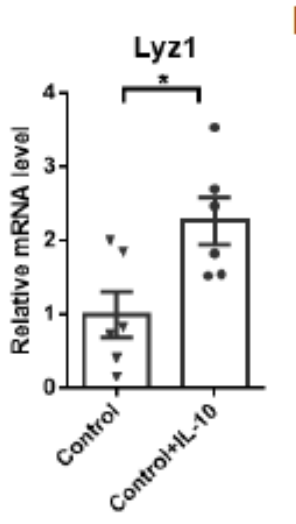

C

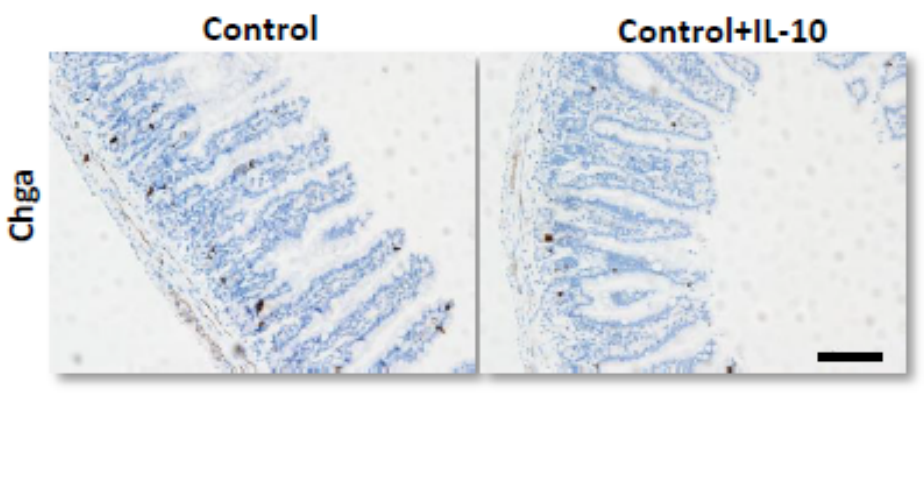

G

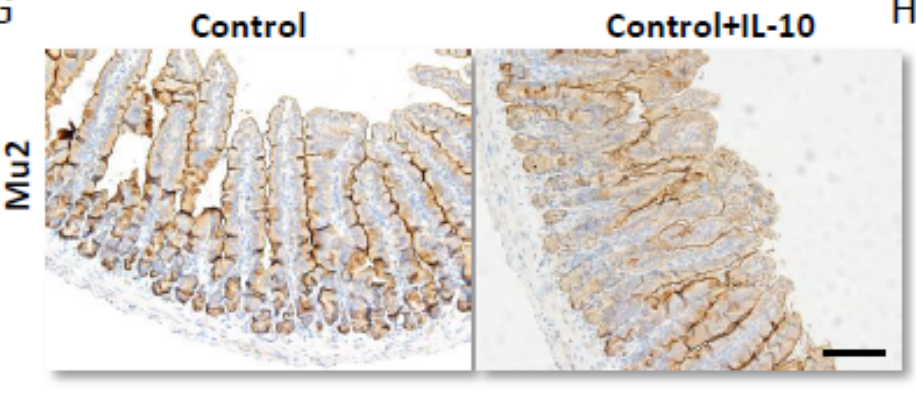

K

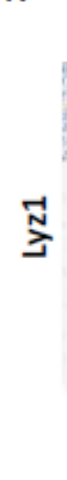

$\mathrm{H}$
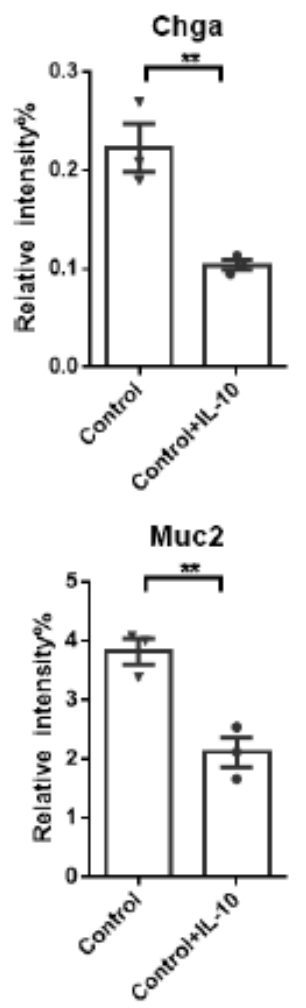

L

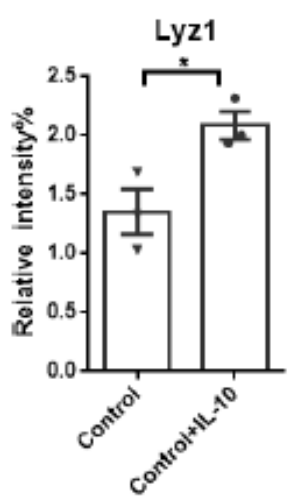

\section{Figure 5}

Interleukin-10 intermingles Epithelial Cell Differentiation. (A) Relative mRNA expression of Chga in the organoids. (B-D) The relative mRNA and protein expression of Chga in the ileum. scale bar, $100 \mu \mathrm{m}$. (E) Relative mRNA expression of Muc2 in the organoids. $(\mathrm{F}-\mathrm{H})$ The relative mRNA and protein expression of

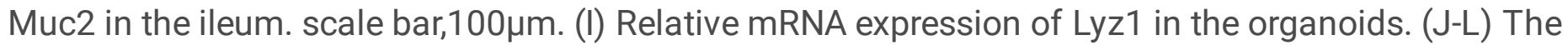
relative mRNA and protein expression of Lyz1 in the ileum. scale bar,100 $\mu \mathrm{m}$. C57BL/6 mice were treated with or without rmlL-10 for 5 days $(n=8)$. Organoids were cultured for 5 days followed by treatment with 
the vehicle or with rmIL-10 $(5 \mathrm{ng} / \mathrm{ml})(\mathrm{n}=6-8)$. The results are expressed as the mean \pm SEM. $\mathrm{n}=8 .{ }^{*} \mathrm{p}<$ $0.05, * \star p<0.01, * \star \star p<0.001$ by two-tailed Student's t test.

\section{Supplementary Files}

This is a list of supplementary files associated with this preprint. Click to download.

- Table1.pdf 